\title{
Autoeficacia: Relación con los hábitos de salud y la calidad de vida en universitarios del noreste de México
}

\section{Self-efficacy: Relationship with health habits and quality of life in university students from northeast Mexico}

\author{
Mayra Lizeth Salgado-Espinosaa , Javier Álvarez Bermúdeza \\ a Facultad de Psicología, Universidad Autónoma de Nuevo León, México
}

\section{Resumen}

Introducción: La autoeficacia es un recurso cognitivo relacionado con el perfil del autocuidado y la evaluación de la satisfacción con la vida de las y los estudiantes. Objetivos: Analizar la diferencia entre el nivel de Autoeficacia General Percibida (AGP) y la práctica o no de hábitos saludables, así como analizar la relación de la AGP con la Calidad de Vida (CV) en los universitarios del noreste de México. Metodología: El diseño fue cuantitativo, correlacional, comparativo, transversal, con una muestra de 300 estudiantes universitarios del noreste de México. Se aplicó el Cuestionario de Hábitos de Salud, la Escala de Autoeficacia General Percibida y la subescala de Satisfacción General con la Vida y Bienestar. Resultados: Se encontró que los universitarios que practicaron actividad física, ejercicio y que no consumen tabaco presentaron niveles más altos de AGP, mientras que las diferencias de los niveles de AGP no fueron estadísticamente significativos, en el cuidado de la alimentación ni en el consumo de alcohol. Además, a mayor AGP mejor CV $(p<.05)$. Conclusiones: La AGP es un factor determinante en la práctica de actividad física y en la prevención del consumo de tabaco, mientras que la alimentación y consumo de alcohol, son comportamientos construidos socialmente.

Palabras clave: Autoeficacia; hábitos de salud; calidad de vida; universitarios; México.

\begin{abstract}
Introduction: Self-efficacy is a cognitive resource related to the profile of self-care and the evaluation of student satisfaction with life. Objectives: To analyze the differences between General Perceived Selfefficacy (GPS-E) and the practice or not of healthy habits, and the relationship between GPS-E and Quality of Life (QoL) in university students of northeastern Mexico. Methodology: The design was quantitative, correlational, comparative, cross-sectional, with a sample of 300 university students from northeastern Mexico. Health Habits Questionnaire, Perceived General Self-Efficacy Scale and General Satisfaction with Life and Wellbeing subscale were applied. Results: It was found that university students who practiced physical activity and exercise and who do not consume tobacco presented higher levels of AGP, while differences in AGP levels were not statistically significant, in the care of food or in the consumption of alcohol. In addition, the higher the AGP the better CV $(p<.05)$. Conclusions: AGP is a determining factor in the practice of physical activity and in the prevention of tobacco consumption, while food and alcohol consumption are socially constructed behaviors.
\end{abstract}

Keywords: Self-efficacy; health habits; quality of life; college students; Mexico. 


\section{Introducción}

a transición a la vida universitaria constituye uno de los principales hitos en el desarrollo del ser humano. Debido a su inherente impacto sobre la adopción y mantenimiento de hábitos de salud, surge como producto del incremento de la autonomía sobre sus pautas de autocuidado, donde la autoeficacia se relaciona como parte de las estrategias de afrontamiento frente a las nuevas condiciones de vida.

En general, los universitarios están condicionados por el entorno en el que se desarrollan y por sus propias experiencias, aspectos que se encuentran en constante evolución y que influyen en su estado de salud (Arora, Shah, Chaturvedi, \& Gupta, 2015; Naciones Unidas, 2013). La autoeficacia es entendida como un factor psicológico relevante para afrontar los obstáculos que se pudieran presentar al practicar comportamientos, saludables o no, debido a su capacidad de tomar decisiones de actuar en determinadas formas respecto a sus hábitos, de acuerdo con su sistema de creencias (Lin, Wood, \& Monterosso, 2015), basado en sus experiencias personales, familiares y educacionales (Pérez \& Álvarez, 2018).

El concepto de la autoeficacia se entiende como un recurso cognitivo que involucra la capacidad del individuo para llevar a cabo conductas para alcanzar logros, basándose en lo que éste espera, adquiriendo resultados distintos (Bandura, 1997). Es así como se ha relacionado la autoeficacia al cuidado de la salud, presentando una gran influencia en las intenciones y los comportamientos relacionados a los hábitos de salud (Schwarzer \& Fuchs, 1995; Olivari \& Urra, 2007), y que podría ser un medio para la necesaria educación para la salud, mejorando la autoeficacia en los hábitos de salud y, de esta forma, mediar el impacto del contexto social (Salgado \& Álvarez, 2018).

Bandura (1997, p.3) definió la autoeficacia como "las creencias en las propias capacidades de organizar y ejecutar los cursos de acción requeridos para producir un objetivo dado". Por lo tanto, la autoeficacia interviene a través de la elección del comportamiento, la cantidad de esfuerzo implementado al cumplimiento de la tarea y la persistencia aun cuando se presentan dificultades, e influye en las habilidades de planeación, automonitoreo y motivación. De esta forma, la autoeficacia toma especial relevancia como un recurso cognitivo relacionado a los hábitos de salud, que sigue las leyes de aprendizaje (Moral \& Álvarez, 2009), debido a que se adquieren a partir de las interacciones sociales; se mantienen porque agilizan el funcionamiento cotidiano; e igualmente, se extinguen, como consecuencia de las evaluaciones cognitivas de practicidad versus beneficio.

De acuerdo a diversos autores (Downes, 2015; Espinoza, Rodríguez, Gálvez, \& MacMillan, 2011; González, Díaz, Mendizabal-Ruiz, Medina, \& Morales, 2014; Martínez et al., 2012; Morales, del Valle, Soto, \& Ivanovic, 2013 ), los comportamientos adoptados con mayor frecuencia durante la etapa universitaria son el alto consumo de alimentos hipercalóricos, la creciente práctica de comportamientos sedentarios (Hidalgo-Rasmussen, Ramírez, \& Hidalgo-San Martin, 2013; Ratner, Hernández, Martel, \& Atalah, 2012) y el consumo de sustancias, tales como alcohol y tabaco (Castañeda \& Romero, 2014).

Salgado, Álvarez, Chávez y Nieto (2017) encontraron que los mayores índices de satisfacción con la vida tienen que ver con actividades relacionadas a la autonomía y libre elección. La autoeficacia sería un factor importante para el manejo de dichas actividades, por lo que en la medida en que el individuo se siente capaz de afrontar los obstáculos que se presentan en relación a sus hábitos de salud, mayor satisfacción con su vida. En este sentido, diversas investigaciones encontraron que los universitarios autoeficaces tienden a evaluar los eventos o demandas como retos (Verdugo-Lucero et al., 2013), incrementando su sentido de participación y su relevancia en sus actividades cotidianas (Cabanach, Valle, Rodríguez, Piñeiro, \& González, 2010), y generando un impacto sobre el bienestar experimentado (Rueda \& Pérez, 2004).

La autoeficacia influye en la evaluación de la calidad de vida, que se utiliza como un indicador subjetivo multidimensional referido al grado de satisfacción con la vida (Salgado, 2018). Según la Organización Mundial de la Salud (OMS, 1997) podemos definir calidad de vida como: "La percepción del individuo de su posición en la vida en el contexto de la cultura y sistemas de valores en los cuales ellos viven y en relación a sus metas, expectativas, estándares y preocupaciones" (p. 1).

En este aspecto, Durán et al. (2012), HidalgoRasmussen, Hidalgo-San Martín, Rasmussen-Cruz y Montaño-Espinoza (2011) y Salgado et al. (2017) reportaron que los universitarios presentan un buen nivel de satisfacción con la vida. En relación con los hábitos de salud, en la investigación de Hidalgo-Rasmussen et al. (2013) se encontró una correlación positiva entre la frecuencia de actividad física y la calidad de vida, debido a que tiene un impacto positivo en la manera de percibir el mundo y de valorar los diferentes ámbitos en los que 
se desarrolla el individuo (Moscoso, Martín, Pedrajas, \& Sánchez, 2013). Por otro lado, Wanden-Berghe et al. (2015) encontró una correlación negativa entre el tiempo invertido en prácticas sedentarias y la calidad de vida.

Por lo tanto, la autoeficacia podría fungir como un mediador entre la adopción y mantenimiento de los hábitos de salud en los universitarios e, igualmente, influir en la calidad de vida, a través de las expectativas generadas de sí mismos.

El objetivo de la presente investigación fue el de identificar los hábitos de salud practicados por los universitarios del noreste de México, analizar la diferencia entre el nivel de AGP y la práctica o no de hábitos saludables, así como analizar la relación de la AGP y la Calidad de Vida en los universitarios del noreste de México.

\section{Métodos}

\section{Diseño}

Para alcanzar el objetivo propuesto se realizó una investigación de tipo cuantitativa, con un alcance descriptivo, correlacional, comparativo, transversal.

\section{Participantes}

Se realizó un muestreo no probabilístico por conveniencia, con una muestra conformada por 300 estudiantes de centros universitarios de la región noreste de México, que comprende a los estados de Coahuila, Nuevo León y Tamaulipas. Participaron 100 estudiantes de Coahuila, 100 de Nuevo León y 100 de Tamaulipas, cuya media de edad fue de 20.53 años (D.E.=2.19), 56\% fueron mujeres y $44 \%$ hombres.

Los criterios de inclusión para la participación en el estudio fueron los siguientes: pertenecer a una institución educativa pública o privada ubicada en Nuevo León, Coahuila y Tamaulipas, tener entre 18 y 24 años, sin encontrarse laborando al momento de la aplicación.

\section{Instrumentos}

Se recopilaron datos sociodemográficos de edad, sexo, carrera y semestre. Se aplicó el Cuestionario de Hábitos de Salud de Álvarez (2004).

Se utilizó la Escala de Autoeficacia General Percibida de Baessler y Schwarzer (1996, citado en Espada, González, Orgilés, Carballo, \& Piqueras, 2012), la cual evalúa la percepción de competencia personal frente a los eventos potencialmente estresantes (Sanjuán, Pérez, y Bermúdez, 2000). La herramienta se compone de
10 preguntas que se puntúan en una escala de cuatro puntos, mostrando las opciones de "cierto", "más bien cierto", "apenas cierto" e "incorrecto", cuyos valores son los siguientes: 4, 3, 2 y 1, respectivamente. En la investigación de Salgado (2018) se obtuvo un $a=.91$.

Para evaluar la calidad de vida, se utilizó el Cuestionario de Calidad de Vida: Satisfacción y Placer de Endicott (2009). Específicamente se usó la subescala de satisfacción general con la vida y bienestar, donde el participante, mediante una escala de seis puntos, con las opciones de "muy insatisfecho", "insatisfecho", "medianamente insatisfecho", "medianamente satisfecho", "satisfecho" y "muy satisfecho", debe evaluar su satisfacción con su estado de salud física, estado de ánimo, las tareas del curso, relaciones sociales y familiares, actividades de tiempo libre, la participación en la vida diaria, situación económica, y la percepción del sentido de vida, donde de acuerdo a Zubara, Foresti, Rossi, Franceschini y Homero (2009) "a mayor puntuación, mayor satisfacción con la vida". Esta subescala presentó un alfa de Cronbach de .94.

\section{Procedimiento}

Para la aplicación de los instrumentos, se acudió a diversos centros universitarios ubicados en los estados Coahuila, Nuevo León y Tamaulipas, para solicitar la autorización de las autoridades. Posteriormente, se realizó la aplicación en las aulas de clase.

\section{Análisis estadístico}

El análisis de datos se realizó mediante el programa SPSS versión 25 , realizando análisis descriptivos para conocer las características de la muestra. Para los análisis comparativos de nivel de AGP y práctica de hábitos de salud, se utilizó el análisis de la $U$ de Mann. Whitney; y el análisis de correlación de Spearman, para evaluar la relación de autoeficacia general percibida y calidad de vida de los universitarios.

\section{Resultados}

En relación con el Índice de Masa Corporal, y basándonos en la clasificación propuesta por la OMS (1995; 2017), el $51 \%$ de los estudiantes presentó un peso normal, mientras que el $31.3 \%$ presentó sobrepeso y el $14.3 \%$ presentó obesidad.

A continuación, se muestran los resultados obtenidos en cuanto a los hábitos de salud practicados en los universitarios del noreste de México. 


\section{Alimentación}

En relación con los hábitos de alimentación, el 75.3\% reportó estar muy dispuesto a cuidar su alimentación, pero solo el $51 \%$ de los participantes consideró que tenía una buena alimentación. Los comportamientos mediante los cuales descuidan su alimentación fueron comiendo comida basura un $45.9 \%$, dejando de comer un $16.3 \%$ y no comiendo a la misma hora un 14.1\% (Figura 1).
Asimismo, las razones por las que no se preocuparon por el cuidado de su alimentación, se relacionaron a la falta de tiempo, en el $35.5 \%$ de los participantes, el $18.9 \%$ por la preferencia hacia la comida chatarra y el $9.2 \%$ porque no ve consecuencias (Figura 2).

El $67.7 \%$ de los estudiantes encuestados preparaba menos de la mitad de los alimentos que consumía en el día, y el $41.8 \%$ reportó que sus padres eran los encargados de la elaboración de los alimentos.

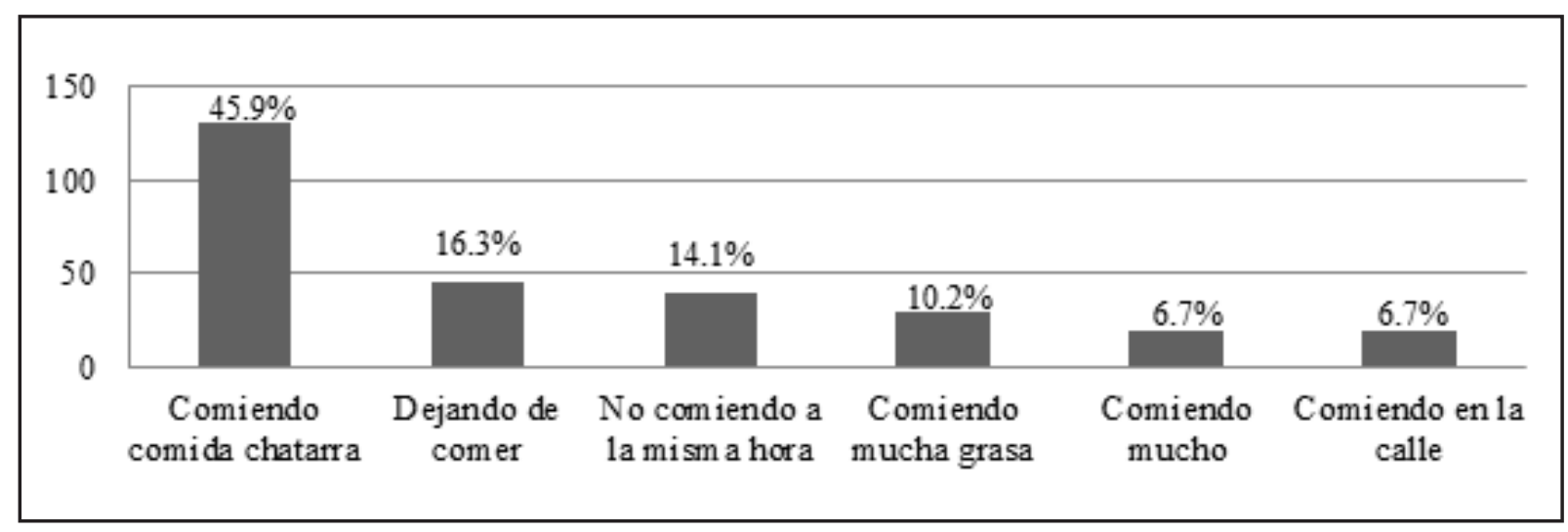

Figura 1. Comportamientos mediante los cuales descuidan su alimentación.

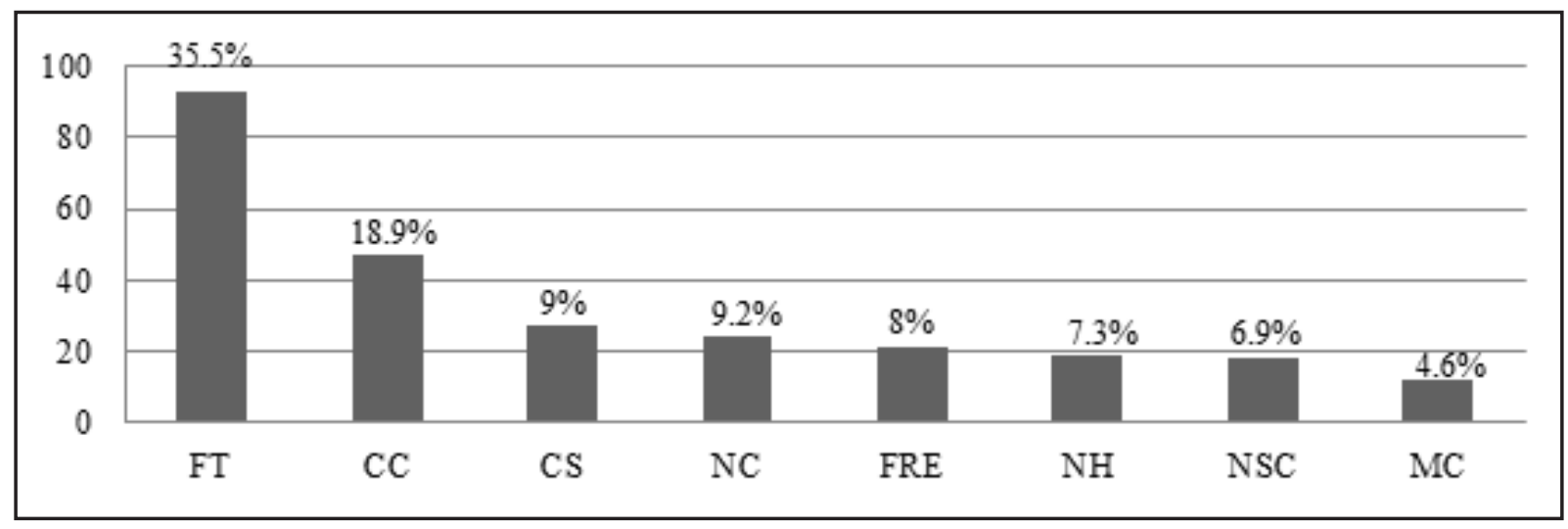

Figura 2. Motivos por los que no les preocupa cuidar su alimentación.

(Nota: FT: falta de tiempo; CC: me gusta la comida chatarra; CS: como lo que sea; NC: porque no veo ninguna consecuencia; FRE: falta de recursos económicos; NH: porque casi no me da hambre; NSC: no sé cómo cuidarla; MC: porque mi mamá la cuida por mí). 


\section{Actividad física y ejercicio}

Respecto a la práctica de actividad física y ejercicio, el $76 \%$ reportó dedicar 1 día o más a la semana a la práctica de actividad física, aunque el $63.6 \%$ no cumplía con la cantidad de ejercicio recomendado para percibir los beneficios.

El 32.2\% de los estudiantes se ejercitaban en casa, el $29.4 \%$ en un gimnasio, el $19.9 \%$ en un parque, el $10.8 \%$ en un club deportivo y el $7.7 \%$ en una escuela. Mientras que en la forma en la que lo practicaban, el $53.8 \%$ lo realizaba en soledad, el $21 \%$ de cualquier forma, el $16.6 \%$ en grupo y el $8.6 \%$ lo realizaba en pareja.

\section{Consumo de tabaco}

El $20 \%$ de los universitarios consumía algo de tabaco, el $14.3 \%$ en un nivel regular, el $3.7 \%$ consumía mucho tabaco y el $2 \%$ demasiado.

La edad de inicio de consumo fue a los 17.02 años (D.E.= 2.39) y consideraron que el principal motivo por el cual una persona fuma era por curiosidad, con una media de 3.56 (D.E.=1.27) y que eran los propios fumadores los responsables de que se inicie con el consumo de tabaco, con una media de 3.87 (D.E.= 1.33) (Tabla 1 y Tabla 2).

Tabla 1. Motivos que llevan a las personas a fumar.

\begin{tabular}{|l|c|c|}
\hline & Media & D.E. \\
\hline Ansiedad & 3.44 & 1.34 \\
\hline Imitación & 3.47 & 1.29 \\
\hline Inseguridad & 2.68 & 1.22 \\
\hline Placer & 3.35 & 1.26 \\
\hline Curiosidad & 3.56 & 1.27 \\
\hline Aparentar ser mayor & 3.31 & 1.36 \\
\hline Costumbre social & 3.36 & 1.24 \\
\hline Modelos sociales & 3.25 & 1.24 \\
\hline Problemas psicológicos y sociales & 3.07 & 1.29 \\
\hline Deseo de evasión & 2.58 & 1.20 \\
\hline
\end{tabular}

Nota: Se evaluó mediante una escala donde 1 equivalía a "totalmente en desacuerdo" y 5 a "totalmente de acuerdo".

Tabla 2. Grado de responsabilidad en el inicio de consumo de tabaco.

\begin{tabular}{|l|c|c|}
\hline & Media & D.E. \\
\hline Los propios fumadores & 3.87 & 1.33 \\
\hline Sus amigos & 3.37 & 2.09 \\
\hline La familia & 3.07 & 1.21 \\
\hline La escuela & 2.84 & 1.19 \\
\hline Los gobiernos & 2.28 & 1.20 \\
\hline La situación económica & 2.31 & 1.22 \\
\hline La publicidad & 3.03 & 1.38 \\
\hline
\end{tabular}

Nota: Se evaluó mediante una escala donde 1 equivalía a "totalmente en desacuerdo" y 5 a "totalmente de acuerdo". 


\section{Consumo de alcohol}

El $36.1 \%$ consumía alcohol de forma regular, el $28.8 \%$ consumía algo de alcohol, el $8 \%$ consumía mucho y el $7 \%$ consumía alcohol en grandes cantidades.

La edad de inicio de consumo de forma usual o repetida fue de 16.99 años (D.E.= 1.98), y las situaciones en la que más lo consumían era en fiestas con sus amigos (73.6\%) y en familia y/o en las comidas (43.5\%) (Figura 3).

Lo reportado anteriormente, correspondió a los resultados del primer objetivo plateado. A continuación, se mostrarán los resultados de los niveles de AGP y los análisis comparativos entre la práctica 0 no de hábitos saludables, que correspondieron al segundo objetivo de la investigación.

\section{Autoeficacia General Percibida y hábitos de salud}

La media de Autoeficacia General Percibida de los estudiantes fue de 29.55 (D.E. = 6.63), por lo que presentan un nivel de autoeficacia media-alta.

Se encontraron diferencias significativas en el grado de autoeficacia general percibida y la práctica de actividad física y ejercicio $(Z=-2.337, p<.05)$, así como en el consumo de tabaco ( $Z=-2.523, p<.05)$, siendo los niveles de autoeficacia más altos en aquellos que tienen hábitos saludables referentes a estos dos comportamientos.

Mientras que no se encontraron diferencias significativas entre los niveles de autoeficacia en los universitarios que tenían una alimentación saludable y los que no la tenían ( $Z=-1.354, p>.05)$, ni tampoco en aquellos que consumían o que no consumían alcohol (Z=-.812, p>.05) (Tabla 3).

Acontinuación, se muestran los análisis concernientes al tercer objetivo de investigación de planteado, que correspondió a identificar la relación entre AGP y la calidad de vida de los universitarios del noreste de México.

\section{Autoeficacia General Percibida y Calidad de vida}

El nivel de calidad de vida fue alto en el $66.7 \%$ de los estudiantes; mientras que el $28.7 \%$ reportó un nivel medio y el $4.7 \%$, un nivel bajo de satisfacción.

De acuerdo con los análisis de correlación de Spearman, se presentó una correlación positiva estadísticamente significativa entre la variable autoeficacia general percibida y la calidad de vida $(r=.454, p<.05)$ (Tabla 4).

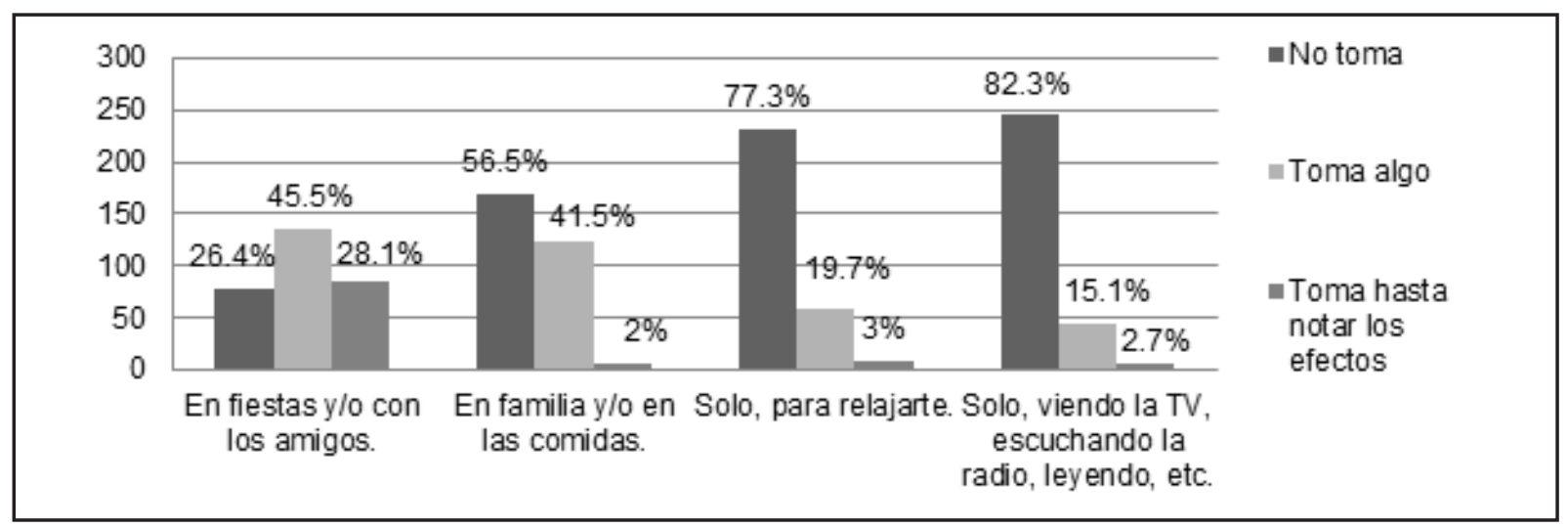

Figura 3. Grado de consumo de alcohol en diferentes situaciones. 
Tabla 3. Prueba de la U de Mann-Whitney de la variable Autoeficacia General Percibida de acuerdo a la práctica de hábitos de salud.

\begin{tabular}{|l|c|c|c|c|c|}
\hline & Respuesta & N & $\begin{array}{c}\text { Rango } \\
\text { Promedio }\end{array}$ & Z & Sig. \\
\hline ¿Tú tienes una buena alimentación? & Sí & 153 & 157.14 & -1.354 & .176 \\
\hline & No & 147 & 143.59 & & \\
\hline ¿Haces ejercicio en forma regular? & Sí & 144 & 162.67 & -2.337 & $.019^{*}$ \\
\hline & No & 156 & 139.27 & & \\
\hline ¿Fumas? & Sí & 120 & 135.05 & -2.523 & $.012^{*}$ \\
\hline & No & 180 & 160.80 & & \\
\hline ¿Consumes alcohol? & Sí & 220 & 147.57 & -.812 & .417 \\
\hline & No & 79 & 156.77 & & \\
\hline
\end{tabular}

${ }^{*} p<.05$

Tabla 4. Análisis de correlación de Spearman entre calidad de vida general y autoeficacia general percibida.

\begin{tabular}{|c|c|c|c|}
\hline & & & Calidad de Vida General \\
\hline Rho de Spearman & $\begin{array}{c}\text { Autoeficacia general } \\
\text { percibida }\end{array}$ & Coeficiente de correlación & $.454^{* *}$ \\
\hline & & Sig. (bilateral) & .000 \\
\hline & & $\mathrm{N}$ & 300 \\
\hline
\end{tabular}

** La correlación es significativa al nivel 0.01 (bilateral).

\section{Discusión}

De acuerdo con los resultados obtenidos, la prevalencia de sobrepeso y obesidad fue de $45.3 \%$, porcentaje notablemente mayor a lo encontrado en la investigación de Moral (2011) en adolescentes escolarizados del noreste de México (23\%); pero menor al porcentaje reportado en la Encuesta Nacional de Salud y Nutrición 2018, en el cual se reportó que el $75.2 \%$ de la población de 20 años o más, presentó sobrepeso y obesidad. Lo anterior podría constituir un importante punto de análisis, ya que visibiliza que a medida que aumenta la edad, la tasa de sobrepeso y obesidad incrementa, lo cual se puede explicar a través de los hábitos que se adquieren y mantienen en la edad adulta.

\section{Alimentación}

En la población universitaria, encontramos que a pesar de que existe una alta intencionalidad para la práctica de una buena alimentación, esto no se ve reflejado en el porcentaje de estudiantes que la cuidaron para el momento de la aplicación de la encuesta. Podemos relacionarlo con los obstáculos que este grupo percibió dentro de los que se encontraron la falta de tiempo, así como a la preferencia de consumo de comida chatarra, y la falta de participación y la alta influencia familiar en la preparación de los alimentos que consumían diariamente.

Dichos factores implicados (falta de tiempo e influencia familiar), destacan aspectos importantes de analizar, tales como las estrategias de administración de tiempo libre, además del cambio de rutina del estudiante, complicando la adopción de un horario establecido para alimentarse, generando una alta preferencia por la comida chatarra, similar a lo encontrado por Milosavljević, Mandić, y Banjari (2015), donde a pesar de tener un valor nutricional deficiente, es accesible económicamente, además de provocar una sensación de saciedad más prolongada.

Mientras que la notoria influencia del contexto familiar en la preparación de alimentos de los estudiantes ha sido reportada anteriormente (ENSANUT MC, 2016), encontrando que la alimentación en familia y la falta de participación en la preparación de alimentación es uno de los principales obstáculos para el cuidado de la 
alimentación, lo que podría relacionarse con lo encontrado por Kramer et al. (2012), quiénes reportaron que el IMC de los jóvenes era mayor cuando los alimentos eran preparados por el cuidador.

\section{Actividad física y ejercicio}

En la práctica de actividad física y ejercicio se puede señalar que, a pesar de que encontramos un porcentaje relativamente alto, un gran porcentaje de ellos no alcanzó la cantidad de 150 minutos a la semana recomendada por la OMS (2017). A partir de esta cantidad de tiempo se empiezan a notar los conocidos beneficios de dicha actividad, tales como la mejora en la capacidad cardiaca y respiratoria, así como también se ha relacionado positivamente el tiempo invertido en la práctica de actividad física con la percepción de un buen estado de salud (Chávez et al., 2018).

Asimismo, es importante señalar que el lugar donde practicaban actividad física y ejercicio era en casa y solos, resultados similares a los reportados previamente en el estudio de Moral et al. (2011) y de Codina y Pestana (2016), lo cual se relaciona con una mayor percepción de autonomía.

\section{Consumo de alcohol}

La mayoría de la población estudiada consumía alcohol, siendo una conducta relacionada con las interacciones sociales con amigos y familia, socialmente aceptado, lo que favorece que sea percibida como una conducta aceptable 0 deseable para formar parte del grupo social (Armendáriz et al., 2014). Asimismo, en la cultura mexicana, el consumo de alcohol es una actividad relacionada a las actividades de tiempo libre, a las festividades, e igualmente, es percibido como un medio para sobrellevar las pérdidas o el proceso de duelo (Alonso \& Álvarez, 2009; Castaño, García, \& Marzo, 2014). De esta forma, tal como lo sostienen Romer y Moreno (2017), el consumo de alcohol está mediado por las creencias positivas respecto a su consumo, por ser una sustancia relacionada al éxito social.

\section{Consumo de tabaco}

Contrario a lo encontrado en torno al consumo de alcohol, el $60 \%$ de los estudiantes universitarios no consumía tabaco, siendo importante resaltar que su consumo lo atribuyeron a factores individuales, como la curiosidad, que podría incrementarse como consecuencia del aumento de las interacciones con población consumidora de tabaco durante la etapa universitaria (Sánchez-Hernández \& Pillon, 2011).

\section{Autoeficacia General Percibida y hábitos de} salud

Respecto a la relación de la AGP y la práctica de hábitos de salud, las similitudes entre los niveles de quienes sí cuidaban su alimentación y los que no llevaban a cabo esta práctica, enfatiza el papel del contexto social inmediato. Los padres, no solamente son responsables de la alimentación de sus hijos universitarios, sino también fungen, de forma implícita, como ejemplos para la adopción y mantenimiento del hábito de la alimentación (Fitzgerald, Heary, Kelly, Nixon, \& Shevlin, 2013), teniendo el potencial de guiar a los universitarios hacia el cuidado de la alimentación.

Similar a lo encontrado en el aspecto de la alimentación, no se presentaron diferencias en un nivel significativo entre la AGP y el consumo de alcohol, lo que puede explicarse por la relación que tiene este comportamiento y el valor social que se le atribuye, donde es percibido como el medio que facilita la integración de los seres humanos a los contextos sociales, siendo relacionado a experiencias placenteras (Gómez, Landeros, Romero, \& Troyo, 2016; Strachan \& Brawley, 2009).

Por otro lado, en relación a la práctica de actividad física, sí se encontraron diferencias significativas entre quienes sí lo realizaban y los universitarios que no lo hacía. Resultados similares a lo reportado por Reigal, Videra y Gil (2014), y relacionándolo con la forma en la que los universitarios reportaron llevarlo a cabo (en casa y solos), podemos destacar que es una actividad que favorece el compromiso personal para el mantenimiento de este hábito (Salgado, 2018).

Igualmente, el nivel de autoeficacia general percibida fue diferente en consumidores y no consumidores de tabaco, siendo más alto en los no fumadores. Al respecto, Bucio (2015) explicó que existe un tipo de autoeficacia que se relaciona con la resistencia, para evitar el consumo de sustancias, y resistir la presión interpersonal o intrapersonal que motiva al consumo, lo cual se relacionaría también a la atribución a factores individuales de la motivación al consumo.

\section{Autoeficacia General Percibida y Calidad de Vida}

Los universitarios presentaron una alta calidad de vida, similar a los encontrado en otras investigaciones (Barraza y Ortiz, 2012; Wanden-Berghe et al., 2015). Lo anterior es relevante porque, de acuerdo a Álvarez (2004) la medida en la que se perciban satisfechos va a ser un factor determinante en la percepción del logro de objetivos a corto, mediano y largo plazo. 
La correlación positiva entre autoeficacia general percibida y calidad de vida, es un indicador relevante para determinar la necesidad de estrategias de intervención enfocadas a las habilidades cognitivas. Según Chavarría y Barra (2014) y Sousa y Lyubomirsky (2001, citado en Reigal et al., 2014), esta percepción de la capacidad para enfrentar los obstáculos que se presenten va a tener su impacto sobre la mejor evaluación de la vida. Se encontró una correlación positiva estadísticamente significativa entre autoeficacia general percibida y calidad de vida, donde dicha relación se puede explicar porque una mejor percepción de la capacidad del individuo para enfrentar los obstáculos que se le presenten tiene como resultado una mejor evaluación de la vida.

\section{Conclusiones}

El estado de salud de los universitarios es el resultado del sinergismo entre los hábitos de salud practicados y sus recursos, tanto materiales como cognitivos. La AGP parece ser un factor protector en hábitos como la práctica de actividad física, a través de las experiencias de éxito generadas y el compromiso personal establecido por este comportamiento, y el no consumo de tabaco, donde la autoeficacia se relaciona con la resistencia a la presión interpersonal que motiva al consumo. Mientras que la autoeficacia no parece tener relación con los hábitos de alimentación y el consumo de alcohol, debido al impacto social sobre estos comportamientos.

Finalmente, la AGP es una habilidad cognitiva importante para la percepción de la calidad de vida. De acuerdo a Plotnikoff et al. (2015, p. 8), con la recién adquirida independencia de los universitarios, "los estudiantes pueden no haber desarrollado habilidades como la autoeficacia, autorregulación y contabilidad, dejándolos en un mayor riesgo de adoptar hábitos no saludables". Por lo tanto, las intervenciones en esta población no solamente deben abordar aspectos informativos de una dieta saludable y la promoción de la práctica de actividad física, sino también promover la adquisición de herramientas que contribuyan a sus habilidades de planeación, auto-monitoreo, incorporando el apoyo social y ambiental para facilitar la adherencia y mantenimiento en comportamientos de salud (Dennis, Potter, Estabrooks, \& Davy, 2012; Kelly et al., 2013), tanto para mejorar el locus de control interno, que se ha relacionado con un IMC normal (Helmer, Krämer, \& Mikolajczyk, 2012), como para afrontar los obstáculos que pudiesen presentarse durante la práctica de hábitos para el cuidado de la salud en la etapa universitaria.

Las principales limitaciones de la presente investigación se encuentran en el tipo de muestreo empleado (no probabilístico por conveniencia). Los resultados no pueden generalizarse a otras poblaciones, por lo que se sugiere realizar un muestreo probabilístico, además de incrementar la cantidad de la muestra, que permita generalizar los datos, así como crear y aplicar un programa de intervención enfocado en mejorar las habilidades de autoeficacia general percibida de los universitarios, como un mediador entre la práctica de hábitos saludables, específicamente de práctica de actividad física y como factor de protección para evitar el consumo de tabaco.

\section{Referencias Bibliográficas}

Alonso, M. M., \& Álvarez, J. (2009). Consumo de alcohol en mujeres adultas: una aproximación cualitativa. En J. Moral \& J. Álvarez. De la salud a la enfermedad. Hábitos tóxicos y alimenticios (pp. 149-192). México: Universidad Autónoma de Nuevo León.

Álvarez, J. (2004). Los jóvenes y sus hábitos de Salud. México, D.F.: Trillas.

Armendáriz, N. A., Alonso, M. M., Alonso, B. A., López, M. A., Rodríguez, L. A., \& Méndez, M. D. (2014). La familia y el consumo de alcohol en estudiantes universitarios. Ciencia y Enfermería, 20(3), 109118.

Arora, S. K., Shah, D., Chaturvedi, S., \& Gupta, P. (2015). Defining and Measuring Vulnerability in Young People. Indian Journal of Community Medicine, 40(3), 193-197. doi: 10.4103/0970-0218.158868

Bandura, A. (1997). Self-efficacy: The exercise of control. New York: Freeman.

Barraza, C. G., \& Ortiz, L. (2012). Factores relacionados a la calidad de vida y satisfacción en estudiantes de enfermería. Ciencia y Enfermería, 18(3), 111-119. doi: 10.4067/S0717-95532012000300011

Bucio, N. (2015). Autoeficacia percibida, resiliencia y consumo de alcoholen estudiantes de pregrado [Universidad Autónoma de Querétaro]. Recuperado de http://ri.uaq.mx/bitstream/123456789/3124/1/ RI003224.pdf

Cabanach, R., Valle, A., Rodríguez, S., Piñeiro, I., \& González, P. (2010). Las creencias motivacionales como factor protector del estrés en estudiantes universitarios. European Journal of Education and Psychology, 3(1), 75-87.

Castañeda, C., \& Romero, S. (2014). Alimentación y 
Consumo de Sustancias (Alcohol, Tabaco y Drogas) del Alumnado Universitario. Análisis en Función del Género y la Práctica de Actividad Físico-Deportiva. Cultura, Ciencia y Deporte, 9 (26), 95-105.

Castaño, G., García, J. A., \& Marzo, J. C. (2014). Consumo de alcohol y factores intervinientes en estudiantes universitarios. Revista Cubana de Salud Pública, 40(1), 47-54.

Chavarría, M. P., \& Barra, E. (2014). Satisfacción vital en adolescentes: relación con la autoeficacia y el apoyo social percibido. Terapia Psicológica, 32(1), 4146. doi: 10.4067/S0718-48082014000100004

Chávez, M. E., Salazar, C. M., Hoyos, G., Bautista, A., González, D., \& Ogarrio, C. E. (2018). Actividad física y enfermedades crónicas no transmisibles de estudiantes mexicanos en función del género. Retos: nuevas tendencias en educación física, deporte y recreación, (33), 169-174.

Codina, N., \& Pestana, J. V. (2016). Actividad físicodeportiva como experiencia de ocio y perspectiva temporal en los jóvenes. Revista de Psicología del Deporte, 25(2), 53-60.

Dennis, E. A., Potter, K. L., Estabrooks, P. A., \& Davy, B. M. (2012). Weight gain prevention for College Freshmen: Comparing two Social Cognitive TheoryBased Interventions with and without Explicit SelfRegulation Training. Journal of Obesity, 2012, 1-10. https://doi.org/10.1155/2012/803769

Downes, L. (2015). Physical Activity and Dietary Habits of College Students. The Journal for Nurse Practitioners, 11(2), 192-200. doi: 10.1016/j. nurpra.2014.11.015

Durán, S., Bazaez, G., Figueroa, K., Berlanga, M., Encina, C., \& Rodríguez, M. (2012). Comparación en calidad de vida y estado nutricional entre alumnos de nutrición y dietética y de otras carreras de la universidad Santo Tomás de Chile. Nutrición Hospitalaria, 27(3), 739746.

Encuesta Nacional de Salud y Nutrición de Medio Camino. (2016). Informe final de resultados. Recuperado de http://promocion.salud.gob.mx/dgps/descargas $1 /$ doctos_2016/ensanut_mc_2016-310oct.pdf

Endicott, J., (2009). Quality of Life Enjoyment and Satisfaction Questionnaire Publications. New York, EUA: Plenum Publishing Corporation.
Espada, J. P., Gonzálvez, M. T., Orgilés, M., Carballo, J. L., \& Piqueras, J. A. (2012). Validación de la escala de autoeficacia general con adolescentes españoles. Electrónico Journal of Research in Educational Psychology, 10(26), 355-370.

Espinoza, L., Rodríguez, F., Gálvez, J., \& MacMillan, N. (2011). Hábitos de alimentación y actividad física en estudiantes universitarios. Revista Chilena de Nutrición, 38(4), 458-465. doi: 10.4067/S071775182011000400009

Fitzgerald, A., Heary, C., Kelly, C., Nixon, E., \& Shevlin, M. (2013). Self-efficacy for healthy eating and peer support for unhealthy eating are associated with adolescents' food intake patterns. Appetite, 63, 3858. doi: 10.1016/j.appet.2012.12.011

Gómez, Z., Landeros, P., Romero, E., \& Troyo, R., (2016). Estilos de vida y riesgos para la salud en una población universitaria. Revista de Salud Pública y Nutrición, 15(2), 9-14.

González, C. E., Díaz, Y., Mendizabal-Ruiz, A. P., Medina, E., \& Morales, J. A. (2014). Prevalencia de obesidad y perfil lipídico alterado en jóvenes universitarios. Nutrición Hospitalaria, 29(2), 315-321. doi: 10.3305/nh.2014.29.2.7054

Helmer, S. M., Krämer, A., \& Mikolajczyk, R. T. (2012). Health-related locus of control and health behaviour among university students in North Rhine Westphalia, Germany. BMC Research Notes, 5, 1-8.

Hidalgo-Rasmussen, C., Hidalgo-San Martín, A., Rasmussen-Cruz, B., y Montaño-Espinoza, R. (2011). Calidad de vida, según percepción y comportamientos de control de peso por género, en estudiantes universitarios adolescentes en México. Cadernos de Saúde Pública, 27(1), 67-77.

Hidalgo-Rasmussen, C., Ramírez, G., y Hidalgo-San Martín, A. (2013). Actividad física, conductas sedentarias y calidad de vida en adolescentes universitarios de Ciudad Guzmán, Jalisco, México. Ciência \& Saúde Coletiva, 18(7), 1943-1952.

Instituto Nacional de Salud Pública \& Instituto Nacional de Estadística y Geografía. (2018). Encuesta Nacional de Salud y Nutrición 2018. Recuperado de https://www.inegi.org.mx/contenidos/programas/ ensanut/2018/doc/ensanut_2018_presentacion_ resultados.pdf 
Kelly, N. R., Mazzeo, S. E., \& Bean, M. K. (2013). Systematic review of dietary interventions with college students: Directions for future research and practice. Journal of Nutrition Education and Behavior, 45(4), 304-313. https://doi.org/10.1016/j. jneb.2012.10.012

Kramer, R. F., Coutinho, A. J., Vaeth, E., Christiansen, K., Suratkar, S., \& Gattelson, J. (2012). Healthier Home Food Preparation Methods and Youth and Caregiver Psychosocial Factors Are Associated with Lower BMI in African American Youth. The Journal of Nutrition, 142(5), 948-954. doi: 10.3945/jn.111.156380

Lin, P., Wood, W., \& Monterosso, J. (2015). Healthy eating habits protect against temptations. Appetite, 1-9. doi: 10.1016/j.appet.2015.11.011

Martínez, M. A., Leiva, A. M., Sotomayor, C., Von Chrismar, A. M., \& Pineda, S. (2012). Factores de riesgo cardiovascular en estudiantes de la Universidad Austral de Chile. Revista Médica de Chile, 140(4), 426-435.

Milosavljević, D., Mandić, M.L., \& Banjari, I. (2015). Nutritional knowledge and dietary habits survey in high school population. Collegium Antropologicum, 39(1), 101-117.

Moral, J. (2011). Aspectos metodológicos de la investigación. En J. Moral, J. Ybarra, J. Álvarez, J. Zapata, \& González, J. Adolescentes escolarizados: sus hábitos de actividad física $y$ alimentación. Un estudio comparativo en el noreste de México (pp. 33-94). México: Fontamara.

Moral, J, \& Álvarez, J. (2009). De la salud a la enfermedad. Hábitos tóxicos y alimenticios (pp. 23-100). México: Universidad Autónoma de Nuevo León.

Moral, J., Ybarra, J., Álvarez, J., Zapata, J., \& González, J. (2011). Adolescentes escolarizados: sus hábitos de actividad física y alimentación. Un estudio comparativo en el noreste de México. México: Fontamara.

Moscoso, D., Martín, M., Pedrajas, N., \& Sánchez, R. (2013). Sedentarismo activo. Ocio, actividad física y estilos de vida de la juventud española. Archivos de Medicina del Deporte, 30(6), 341-347.

Naciones Unidas. (2000). Resolution Adopted by the General Assembly 54/120 Policies and programmes involving youth. Recuperado de:http:/l
www.un.org/en/ga/search/view_doc.asp?symbol=A/ $\mathrm{RES} / 54 / 120$

Olivari, C., \& Urra, E. (2007). Autoeficacia y conductas de salud. Ciencia y Enfermería, 13(1), 9-15.

Organización Mundial de la Salud. (1995). Physical status: the use and interpretation of anthropometry (p.452). Recuperado de http:// apps.who.int/iris/bitstream/10665/37003/1/WHO_ TRS_854.pdf

Organización Mundial de la Salud. (1997). WHOQOL: Measuring Quality of Life. Recuperado de https:// www.who.int/healthinfo/survey/whoqol-qualityoflife/en/

Organización Mundial de la Salud. (2017). Sobrepeso y obesidad. Recuperado de: http://www.who.int/ mediacentre/factsheets/fs311/es/

Pedraza, B. D. L. A. P., \& Álvarez, J. (2018). Representations of eating habits: Differences between pre-adolescents and their parents. Papers on Social Representations, 27(2), 4.1-4.23.

Ratner, R., Hernández, P., Martel, J., \& Atalah, E. (2012). Calidad de la alimentación y estado nutricional en estudiantes universitarios de 11 regiones de Chile. Revista Médica de Chile, 140, 1571-1579.

Reigal, R., Videra, A., \& Gil, J. (2014). Práctica física, autoeficacia general y satisfacción vital en la adolescencia. Revista Internacional de Medicina y Ciencias de la Actividad Física y el Deporte, 14(55), 561-576.

Rojas, M. A. (2014). Nivel de autoeficacia de los empleados de la Confederación Deportiva Autónoma de Guatemala. (Tesis de Licenciatura, Universidad Rafael Landívar, Guatemala). Recuperada de http://biblio3.url.edu.gt/ Tesario/2014/05/43/Rojas-Manuel.pdf

Romer, D., \& Moreno, M. (2017). Digital Media and Risks for Adolescent Substance Abuse and Problematic Gambling. Pediatrics, 140(Supl. 2), 102-106. doi: 10.1542/peds.2016-1758L

Rueda, B., \& Pérez, A. (2004). Personalidad y percepción de autoeficacia: influencia sobre el bienestar y el afrontamiento de los problemas de salud. Revista de Psicopatología y Psicología Clínica, 9(3), 205-219.

Salgado, M. L. (2018). Relación de los hábitos de salud, la imagen corporal y la autoeficacia 
con la calidad de vida en los universitarios del noreste de México. (Tesis de Maestría, Universidad Autónoma de Nuevo León, México). Recuperado de http://eprints.uanl.mx/16383/

Salgado, M. L., \& Álvarez, J. (2018). Imagen corporal en universitarios del noreste de México: estudio comparativo por sexo. Revista de Psicología de la Salud, 6(1), 58-86.

Salgado, M. L., Álvarez, J., Nieto, D., \& Chávez, M. (2017). Comparación de la calidad de vida en estudiantes universitarios de Cataluña, Coahuila y Nuevo México. Revista de Psicología de la Salud, 5(1), 1-27.

Sánchez-Hernández, C. M., \& Pillon, S. C. (2011). Tabaquismo entre universitarios: caracterización del uso en la visión de los estudiantes. Revista Latinoamericana de Enfermagem, 19, 730-737.

Sanjuán, P., Pérez, A., y Bermúdez, J. (2000). Escala de autoeficacia general: datos psicométricos de la adaptación para población española. Psicothema, 12(2), 509-513.

Schwarzer, R., \& Fuchs, R. (1995). Self-efficacy and Health Behavior. Recuperado de: http://userpage. fu-berlin.de/ gesund/publicat/conner9.htm

Strachan, S. M., \& Brawley, L. R. (2009). Healthy-eater identity and self-efficacy predict healthy eating behavior. Journal of Health Psychology, 14(5), 684-695. doi: 10.1177/1359105309104915

Verdugo-Lucero, J., Ponce, B., Guardado-Llamas, R., Meda-Lara, R., Uribe-Alvarado, J., \& GuzmánMuñiz, J. (2013). Estilos de afrontamiento al estrés y bienestar subjetivo en adolescentes y jóvenes. Revista Latinoamericana de Ciencias Sociales, Niñez y Juventud, 11(1), 79-91. Doi: doi: 10.11600/1692715x.1114120312

Wanden-Berghe, C., Martín-Rodero, H., RodríguezMartín, A., Novalbos-Ruiz, J., Martínez, E., SanzValero, J., ...Irles, J. (2015). Calidad de vida y sus factores determinantes en universitarios españoles de Ciencias de la Salud. Nutrición Hospitalaria, 31(2), 952-958.

Zubara, C., Foresti, K., Rossi, M., Franceschini, P., \& Homero, W. (2009). Portuguese versión of the Quality of Life Enjoyment and Satisfaction Questionnaire: a validation study. Revista Panameña de Salud Pública, 25(5), 443-448. 